\title{
Relapsing Polychondritis With Initial Presentations of Recurrent Negative-Pressure Pulmonary Edema and Acute Respiratory Failure
}

\author{
Meng-Fang Wu, Yi-Shan Li, Chen-Yiu Hung MD, Wei-Chieh Chao MD, Zhey-Ying Fu MD, \\ Kuo-Chin Kao MD, Chung-Chi Huang MD, Ying-Huang Tsai MD, and Meng-Jer Hsieh MD
}

\begin{abstract}
Relapsing polychondritis is a rare autoimmune disease causing inflammation in cartilaginous structures and other tissues throughout the body. Negative-pressure pulmonary edema (NPPE) due to laryngeal swelling from relapsing polychondritis is rare and has not been reported. Here, we report a case of relapsing polychondritis in an 18-y-old female who presented with recurrent NPPE and acute respiratory failure, which was diagnosed initially as ARDS during the influenza season. She underwent an emergent tracheotomy to relieve the upper airway obstruction resulting from severe laryngeal edema. A chest radiograph showed diffuse infiltrations, pneumothorax, and pneumomediastinum. The pulmonary infiltrations resolved rapidly in $2 \mathrm{~d}$, and NPPE was diagnosed. Left ear swelling with erythematous change and saddle nose developed during the course of hospitalization, and an ear biopsy demonstrated severe cartilage necrosis. Relapsing polychondritis was diagnosed based on clinical images and pathological findings. Key words: relapsing polychondritis; negativepressure pulmonary edema; acute respiratory failure; barotrauma. [Respir Care 2015;60(5):e101-e104. (C) 2015 Daedalus Enterprises]
\end{abstract}

\section{Introduction}

Relapsing polychondritis is an immune-mediated disease of unknown etiology with inflammation of cartilaginous structures and other tissues throughout the body. ${ }^{1}$ Negative-pressure pulmonary edema (NPPE) typically re-

Ms Wu and Drs Kao and Huang are affiliated with the Department of Respiratory Therapy; Drs Hung, Fu, Kao, and Huang are affiliated with the Department of Pulmonary and Critical Care Medicine; and Dr Chao is affiliated with the Department of Otolaryngology, Linkou Chang-Gung Memorial Hospital, Chang-Gung Medical Foundation, Taoyuan County, Taiwan. Ms Li and Drs Tsai and Hsieh are affiliated with the Department of Respiratory Therapy, and Drs Tsai and Hsieh are also affiliated with the Department of Pulmonary and Critical Care Medicine, Chiayi ChangGung Memorial Hospital, Chang-Gung Medical Foundation, Chiayi County, Taiwan. Drs Kao, Huang, Tsai, and Hsieh are also affiliated with the Department of Respiratory Therapy, Chang-Gung University, Taoyuan County, Taiwan.

The authors have disclosed no conflicts of interest.

Correspondence: Meng-Jer Hsieh MD, Department of Pulmonary and Critical Care Medicine, Chiayi Chang-Gung Memorial Hospital, ChangGung Medical Foundation, No. 6, West Section, Jiapu Road, Puzi City, Chiayi County 613, Taiwan. E-mail: mengjer@yahoo.com.

DOI: $10.4187 /$ respcare. 03274 sults from acute upper airway obstruction such as laryngospasm during the perioperative period. ${ }^{2}$ NPPE resulting from relapsing polychondritis has not been reported in the literature. Here, we report the case of an 18-y-old female with relapsing polychondritis showing initial signs of recurrent NPPE and hypoxemic respiratory failure.

\section{Case Report}

The patient was an 18-y-old female who was generally healthy and without prior systemic disease. She was intubated due to acute respiratory failure in a community hospital and transferred to our hospital for ARDS related to suspected influenza pneumonitis. The progression of influenza pneumonitis into ARDS is not uncommon in the influenza season in Taiwan, but influenza A or B virus was not detected in an oropharyngeal swab by reverse transcription polymerase chain reaction. The initial chest radiograph and computed tomography showed diffuse pulmonary infiltrations, subcutaneous emphysema, and pneumomediastinum, but no identifiable pneumothorax (Fig. 1, $\mathrm{A}$ and $\mathrm{B})$. Pressure controlled continuous mandatory ventilation (PC-CMV) was administered with a peak airway pressure of $36 \mathrm{~cm} \mathrm{H}_{2} \mathrm{O}$ and a PEEP of $12 \mathrm{~cm} \mathrm{H}_{2} \mathrm{O}$. The tidal volume was $430 \mathrm{~mL}$, and her breathing frequency was 


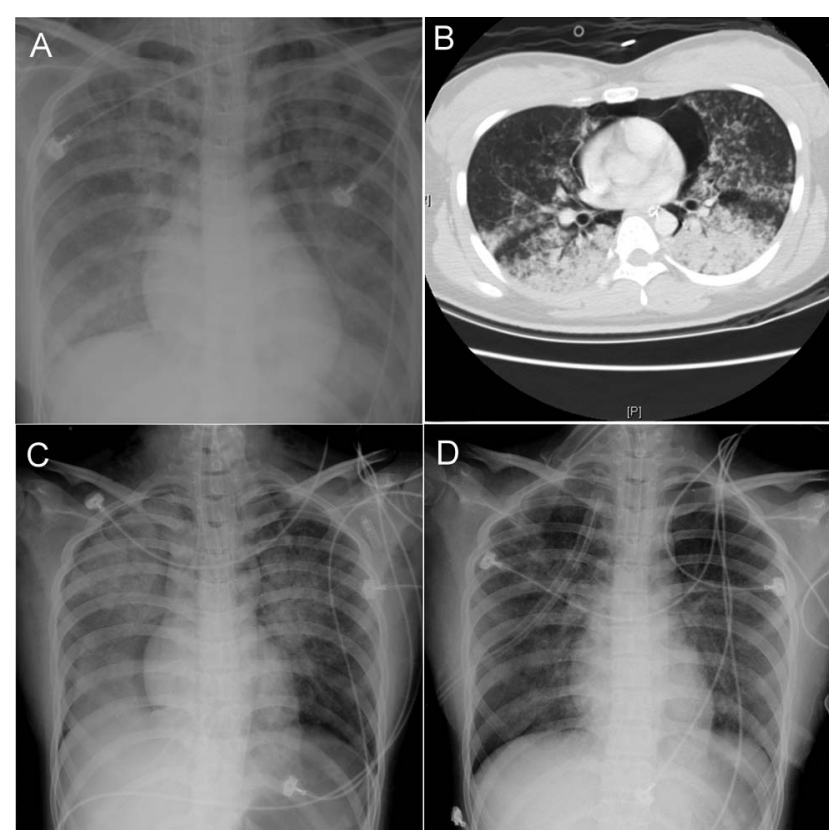

Fig. 1. A: On the first admission, chest radiograph revealed diffuse bilateral pulmonary infiltrations. B: chest computed tomography showed consolidations over bilateral dependent parts of the lungs, consistent with pulmonary edema. Pneumomediastinum was also identified by chest computed tomography. C: Chest radiograph on the day of tracheotomy showed bilateral pulmonary infiltrations with perihilar distribution, mild pneumomediastinum, and rightsided pneumothorax. D: Chest radiograph $2 \mathrm{~d}$ post-tracheotomy showed significant improvement of the infiltrations bilaterally.

31 breaths/min. The $\mathrm{P}_{\mathrm{aO}_{2}}$ was $67.4 \mathrm{~mm} \mathrm{Hg}$ with an $\mathrm{F}_{\mathrm{IO}_{2}}$ of 1.0 on the first day of admission. The level of PC-CMV was decreased soon after admission, and $5 \mathrm{~d}$ after the initial admission, the ventilator mode was changed to pressure support with an $\mathrm{F}_{\mathrm{IO}_{2}}$ of 0.35 . Mechanical ventilation was discontinued, and she was extubated $8 \mathrm{~d}$ later. She was treated with parenteral piperacillin with tazobactam, teicoplanin, and clarithromycin for suspected pneumonia. Initial blood cultures were negative for any bacteria, and an endotracheal aspiration culture grew rare Staphylococcus aureus only. Antibiotics were discontinued soon after she was transferred to the ward. Postextubation stridor developed, and vocal cord edema, initially believed to be related to the previous intubation, was discovered by laryngoscopy. She was discharged after 3 weeks of hospitalization without respiratory distress. Because her mother reported a nocturnal wheeze, inhaled fluticasone $(100 \mu \mathrm{g})$ with salmeterol $(50 \mu \mathrm{g})$ in a single inhaler was given at one inhalation twice daily.

Two weeks after she was discharged from our hospital, she returned to the emergency room with cough, sore throat, rhinorrhea, nasal obstruction, and hyperthermia. Her body temperature was $36.5^{\circ} \mathrm{C}$, her blood pressure was $128 / 86 \mathrm{~mm} \mathrm{Hg}$ with a pulse rate of 117 beats/min, and



Fig. 2. A: Transverse section of head and neck computed tomography at the level of cricoid cartilage showed deformity of the cricoid cartilage (black arrow), laryngeal edema (white arrow), and airway narrowing. B: Sagittal section of head and neck computed tomography showed saddle nose (white arrow), subglottic swelling (black arrow), and airway narrowing. C: Bronchoscopy 1 week post-tracheotomy revealed unresolved subglottic narrowing and mild vocal cord swelling.

her breathing frequency was 21 breaths/min. On physical examination, tachypnea, breath sounds with wheezes, and tachycardia were present. The chest radiograph was normal at that time. However, dyspnea with stridor developed $3 \mathrm{~d}$ later, and laryngoscopy again revealed vocal cord edema. These conditions improved after treatment with dexamethasone administered intravenously at $5 \mathrm{mg}$ every $8 \mathrm{~h}$ and epinephrine administered by inhalation at $1 \mathrm{mg} 3$ times daily. Computed tomography showed saddle nose and deformity at the posterior rim of the cricoid cartilage, laryngeal edema, or laryngitis with compromised airway (Fig. 2, A and B). Bronchoscopy revealed subglottic narrowing, which could be related to underlying cartilage inflammation and edema. Left ear swelling with erythematous changes associated with hearing impairment developed during this period, but pure tone audiometry was normal 1 week before the second ICU admission.

While in the ward, her sister noted the patient's respiratory distress with central cyanosis. Marked laryngeal swelling with nearly total occlusion of the upper airway was found during endotracheal intubation. An emergent tracheotomy was performed at bedside. She regained consciousness after the artificial airway was created and she was mechanically ventilated.

The $\mathrm{S}_{\mathrm{aO}_{2}}$ was $92 \%$ with $\mathrm{PC}-\mathrm{CMV}$ and an $\mathrm{F}_{\mathrm{IO}_{2}}$ of 1.0 when the patient was transferred to the ICU. The peak airway pressure was $30 \mathrm{~cm} \mathrm{H}_{2} \mathrm{O}$, and the PEEP was $6 \mathrm{~cm} \mathrm{H}_{2} \mathrm{O}$. The tidal volume was $400 \mathrm{~mL}$, and her breathing frequency was 25 breaths/min. Upon recovering from sedation for the tracheotomy, she became irritable and was not breathing in synchrony with the ventilator. She was sedated and paralyzed with midazolam and cisatracurium infusion, which was discontinued the next day. A chest radiograph post-tracheotomy revealed bilateral pulmonary infiltrations with prominent left-sided perihilar infiltration, pneumomediastinum, and right-sided pneumothorax (see Fig. 1C). Pink frothy sputum was found in the endotracheal tube, and inspiratory crackles were auscultated in 


\section{Relapsing Polychondritis With Recurrent NPPE and Acute Respiratory Failure}

both lower lungs. The PEEP was increased from 6 to $10 \mathrm{~cm} \mathrm{H}_{2} \mathrm{O}$ after insertion of a chest tube for drainage.

A follow-up chest radiograph $2 \mathrm{~d}$ later showed marked improvement of the bilateral infiltrates (see Fig. 1D), and the $\mathrm{F}_{\mathrm{IO}_{2}}$ was decreased to 0.35 with adequate $\mathrm{S}_{\mathrm{aO}_{2}}$. An echocardiograph post-tracheotomy revealed adequate left ventricle systolic function without impaired wall motion, which indicated that the pulmonary edema was not directly related to heart disease. Empirical antibiotics with teicoplanin and ciprofloxacin had been prescribed in the ICU, but the endotracheal aspiration culture grew mixed flora without an identifiable bacterial pathogen. The above clinical features led to the diagnosis of NPPE related to acute upper airway obstruction. Dexamethasone was continued intravenously at $5 \mathrm{mg}$ every $8 \mathrm{~h}$ in the ICU. Bronchoscopy 1 week post-tracheotomy revealed unresolved subglottic narrowing and mild vocal cord swelling (see Fig. 2B). Mechanical ventilation was discontinued $7 \mathrm{~d}$ post-tracheotomy. The saddle nose became more obvious after she was transferred from the ICU to a ward. A left ear biopsy revealed necrotizing chondritis with chronic inflammation and necrosis. These clinical and pathological findings fulfilled the McAdam et $\mathrm{al}^{3}$ criteria for diagnosing relapsing polychondritis. The patient was discharged with oral methylprednisolone $(28 \mathrm{mg} / \mathrm{d})$ and was monitored at rheumatology clinics in a stable condition. The maintenance dosage of oral methylprednisolone was tapered gradually to $10 \mathrm{mg} / \mathrm{d}$, and she still has the tracheostomy to date.

\section{Discussion}

Relapsing polychondritis is an autoimmune disorder that targets cartilaginous and some non-cartilaginous tissues. The etiology of relapsing polychondritis remains unknown. It is a rare disease with an estimated incidence of $3 / 1,000,000$, and onset usually occurs between 40 and $60 \mathrm{y}$ of age. ${ }^{4}$ The most common targeted organ is the ear. Other organs, including large airways, nose, eyes, joints, and heart, may also be targeted. ${ }^{1}$ McAdam et $\mathrm{al}^{3}$ defined the criteria for diagnosis of relapsing polychondritis as the presence of any 3 of the following 6 clinical presentations: (1) bilateral auricular chondritis, (2) non-erosive seronegative inflammatory arthritis, (3) nasal chondritis, (4) ocular inflammation, (5) respiratory tract chondritis, and (6) audiovestibular damage. The overall survival rate was $70 \%$ at $4 \mathrm{y}$ in 1976 and increased to $94 \%$ at $8 \mathrm{y}$ in 1998.5

The lower respiratory tract is affected in $20-50 \%$ of patients with relapsing polychondritis, with clinical presentations of subglottic stenosis, tracheal stenosis, tracheal wall thickening, or tracheobronchomalacia. ${ }^{6}$ There are 3 stages of airway obstruction: (1) airway stenosis due to inflammatory swelling in the active stage, (2) progressive destruction of the respiratory tract cartilage causing dynamic collapse of the airway in the early stage, and (3) formation fibrous tissue causing contraction in the late stage. ${ }^{7}$ Our patient was in the first disease stage, showing upper airway inflammatory swelling and narrowing. Respiratory symptoms, including dyspnea, cough, chest discomfort, husky voice, and stridor, can be signs of airway involvement. ${ }^{6}$ Most patients with airway manifestations are stabilized with supportive treatment. ${ }^{8}$ Noninvasive ventilation could be a treatment of choice for airway involvement in relapsing polychondritis to keep the narrowed airways from collapsing. ${ }^{9}$ In the case we reported, the obstruction was so severe as to result in acute respiratory failure and NPPE. The laryngeal edema and swelling did not resolve completely even with steroid treatment after discharge. An artificial airway creation to bypass this obstruction was indicated.

NPPE or postobstructive pulmonary edema resulting from acute upper airway obstruction is a potentially fatal condition. The large negative intrathoracic pressure generated by forced inspiration against an obstructed airway is thought to be the principal mechanism. ${ }^{10}$ The inspiratory efforts to overcome the upper airway obstruction generate highly negative intrapleural and intrapulmonary pressures. The resulting high pressure gradient causes an increase in venous return and blood flow to the right side of the heart and increased left heart afterload. ${ }^{11}$ This combination leads to an increase in hydrostatic pressures and pulmonary edema. ${ }^{12}$ Two subclasses of NPPE have been described: type I is associated with forceful inspiratory effort against an acute airway obstruction, and type II develops after relief of a chronic partial airway obstruction. ${ }^{12}$ The most common etiology of type I NPPE is laryngospasm during intubation or after anesthesia, accounting for as much as $50 \%$ of adult cases. ${ }^{2}$ Other common causes of type I NPPE include epiglottitis, croup, choking with foreign body, strangulation, hanging, endotracheal tube obstruction, laryngeal tumor, goiter, postoperative vocal cord paralysis, and near drowning. ${ }^{2}$ In our patient, type I NPPE was caused by laryngeal edema, which resulted from relapsing polychondritis.

The first priority for management of NPPE is to relieve the airway obstruction and correct hypoxemia. Mechanical ventilation with PEEP and a high $\mathrm{F}_{\mathrm{IO}_{2}}$ may be necessary in patients with severe NPPE. Diuretics are used to treat cardiogenic pulmonary edema, but may result in hypovolemia in NPPE patients without fluid overload and should thus be used cautiously.

NPPE caused by acute upper airway obstruction resulting from relapsing polychondritis has not been reported in the literature. In our case, NPPE was the first presentation of relapsing polychondritis and developed before the occurrence of auricular chondritis and saddle nose. Initially, respiratory failure was mistakenly assumed to be related to influenza infection during the influenza season. The correct diagnosis was made after the typical ear and nose abnormalities developed. With a more clear clinical course on the second admission, she experienced mild fever, sore throat, 


\section{Relapsing Polychondritis With Recurrent NPPE and Acute Respiratory Failure}

rhinorrhea, and cough before the development of respiratory distress. These symptoms were consistent with an episode of viral upper respiratory tract infection. It is possible that a viral infection could induce activation of relapsing polychondritis.

Spontaneous pneumomediastinum has been reported in a patient with NPPE, ${ }^{13}$ and pneumothorax has occurred in a patient with relapsing polychondritis. ${ }^{14}$ Our patient became irritable and was not breathing in synchrony with the ventilator after recovering from the sedation given before the tracheotomy. Later, she experienced not only pneumothorax but also pneumomediastinum and subcutaneous emphysema, which most likely resulted from barotrauma with positive-pressure mechanical ventilation. Similarly, the pneumomediastinum discovered by chest computed tomography on the first ICU admission might be also related to barotrauma.

Relapsing polychondritis is a rare autoimmune disease. Without typical signs and symptoms, it can be easily misdiagnosed. This case initially presented to us with serious but atypical signs of NPPE, a rare presentation and a potentially fatal complication in relapsing polychondritis, leading to delayed diagnosis. The NPPE associated with acute respiratory failure and complicated by pneumomediastinum and pneumothorax was successfully managed with tracheotomy, chest tube drainage, and mechanical ventilation with PEEP. Tracheal and bronchial narrowing is the most serious complication of this disease and should be managed carefully.

\section{REFERENCES}

1. Lahmer T, Treiber M, von Werder A, Foerger F, Knopf A, Heemann U, Thuermel K. Relapsing polychondritis: an autoimmune disease with many faces. Autoimmun Rev 2010;9(8):540-546.
2. Louis PJ, Fernandes R. Negative pressure pulmonary edema. Oral Surg Oral Med Oral Pathol Oral Radiol Endod 2002;93(1):4-6.

3. McAdam LP, O'Hanlan MA, Bluestone R, Pearson CM. Relapsing polychondritis: prospective study of 23 patients and a review of the literature. Medicine 1976;55(3):193-215.

4. Kent PD, Michet CJ Jr, Luthra HS. Relapsing polychondritis. Curr Opin Rheumatol 2004;16(1):56-61.

5. Yoo JH, Chodosh J, Dana R. Relapsing polychondritis: systemic and ocular manifestations, differential diagnosis, management, and prognosis. Semin Ophthalmol 2011;26(4-5):261-269.

6. Rafeq S, Trentham D, Ernst A. Pulmonary manifestations of relapsing polychondritis. Clin Chest Med 2010;31(3):513-518.

7. Mohsenifar Z, Tashkin DP, Carson SA, Bellamy PE. Pulmonary function in patients with relapsing polychondritis. Chest 1982;81(6): 711-717.

8. Sarodia BD, Dasgupta A, Mehta AC. Management of airway manifestations of relapsing polychondritis. Chest 1999;116(6):16691675.

9. Ishikawa S, Yamazaki M, Takei Y, Miyazaki A, Hanyu N. A case of relapsing polychondritis with oculobulbar symptoms and successful treatment of respiratory failure with BiPAP. Rinsho Shinkeigaku 1999;39(10):1040-1044.

10. Bhaskar B, Fraser J. Negative pressure pulmonary edema revisited: pathophysiology and review of management. Saudi J Anaesth 2011; 5(3):308-313.

11. Lang SA, Duncan PG, Shephard DA, Ha HC. Pulmonary oedema associated with airway obstruction. Can J Anesth 1990;37(2):210218.

12. Udeshi A, Cantie SM, Pierre E. Postobstructive pulmonary edema. J Crit Care 2010;25(3):508.e1-508.e5.

13. Fiorelli A, Brongo S, D'Andrea F, Santini M. Negative-pressure pulmonary edema presented with concomitant spontaneous pneumomediastinum: Moore meets Macklin. Interact Cardiovasc Thorac Surg 2011;12(4):633-635.

14. Wu S, Sagawa M, Suzuki S, Kumagai-Braesch M, Honda Y, Sato M, Kondo T. Pulmonary fibrosis with intractable pneumothorax: new pulmonary manifestation of relapsing polychondritis. Tohoku J Exp Med 2001;194(3):191-195. 